\title{
Observations of the stratorotational instability in rotating concentric cylinders
}

\author{
Ruy Ibanez and Harry L. Swinney* \\ Center for Nonlinear Dynamics and Physics Department, University of Texas at Austin, \\ Austin, Texas 78712, USA \\ Bruce Rodenborn ${ }^{\dagger}$ \\ Physics Program, Centre College, Danville, Kentucky 40422, USA \\ (Received 10 April 2016; published 1 September 2016)
}

\begin{abstract}
We study the stability of density stratified flow between corotating vertical cylinders with rotation rates $\Omega_{o}<\Omega_{i}$ and radius ratio $r_{i} / r_{o}=0.877$, where subscripts $o$ and $i$ refer to the outer and inner cylinders. Just as in stellar and planetary accretion disks, the flow has rotation, anticyclonic shear, and a stabilizing density gradient parallel to the rotation axis. The primary instability of the laminar state leads not to axisymmetric Taylor vortex flow but to a nonaxisymmetric stratorotational instability (SRI). The present work extends the range of Reynolds numbers and buoyancy frequencies $[N=\sqrt{(-g / \rho)(\partial \rho / \partial z)}]$ examined in previous experiments. We present the first experimental results for the axial wavelength $\lambda$ of the instability as a function of the internal Froude number, $\mathrm{Fr}=\Omega_{i} / N ; \lambda$ increases by nearly an order of magnitude over the range of Fr examined. For small outer cylinder Reynolds number, the SRI occurs for inner inner Reynolds number larger than for the axisymmetric Taylor vortex flow (i.e., the SRI is more stable). For somewhat larger outer Reynolds numbers the SRI occurs for smaller inner Reynolds numbers than Taylor vortex flow and even below the Rayleigh stability line for an inviscid fluid. Shalybkov and Rüdiger [Astron. Astrophys. 438, 411 (2005)] proposed that the laminar state of a stably stratified rotating shear flow should be stable for $\Omega_{o} / \Omega_{i}>r_{i} / r_{o}$, but we find that this stability criterion is violated for $N$ sufficiently large. At large Reynolds number the primary instability is not the SRI but a previously unreported nonperiodic state that mixes the fluid.
\end{abstract}

DOI: 10.1103/PhysRevFluids.1.053601

\section{INTRODUCTION}

Stellar and planetary accretion disks that have Keplerian or quasi-Keplerian velocity profiles $\Omega_{\phi}(r) \propto r^{-3 / 2}$ are a canonical example of a rotating flow with anticyclonic shear. Physical and experimental models of accretion disks focus on understanding the mechanisms by which angular momentum is transported radially outward to allow collapse of the central object and planetary bodies. These mechanisms broadly include magneto-hydrodynamic and purely hydrodynamic processes [1], but studies of concentric rotating cylinder systems have led to a debate whether hydrodynamic processes alone make significant contributions to the angular momentum transport [2,3]. However, many accretion disks include cold regions where the effects of magnetic fields are minimal and the hydrodynamic contribution to the angular momentum transport via

*swinney@chaos.utexas.edu

†bruce.rodenborn@centre.edu

Published by the American Physical Society under the terms of the Creative Commons Attribution 3.0 License. Further distribution of this work must maintain attribution to the author(s) and the published article's title, journal citation, and DOI. 
TABLE I. Experimental parameters.

\begin{tabular}{lll}
\hline \hline & Concentric rotating cylinder system & \\
\hline Inner radius: & Outer radius: & Height: \\
$r_{i}=4.218 \mathrm{~cm}$ & $r_{o}=4.811 \mathrm{~cm}$ & $h=25.6 \mathrm{~cm}$ \\
Radius ratio: & Gap width: & Aspect ratio: \\
$\eta=r_{i} / r_{o}=0.877$ & $d=r_{o}-r_{i}=0.593 \mathrm{~cm}$ & $\Gamma=h / d=43.4$ \\
Inner rotation rate: & Outer rotation rate: & Kinematic viscosity: \\
$\Omega_{i}$ & $\Omega_{o}$ & $v$ \\
\hline & \multicolumn{1}{c}{ Control parameters } & \\
\hline Inner Reynolds number: & Outer Reynolds number: & Buoyancy frequency: \\
$\operatorname{Re}_{i}=\left(\Omega_{i} r_{i}\right) d / v$ & $\operatorname{Re}{ }_{o}=\left(\Omega_{o} r_{o}\right) d / v$ & $N=\sqrt{-\frac{g}{\rho} \frac{d \rho}{d z}}$ \\
$\operatorname{Rotation~ratio:~}_{\mu=\Omega_{o} / \Omega_{i}}$ & $\mathrm{Internal} \mathrm{Froude} \mathrm{number}[8,9]:$ & $q$ parameter $[1]:$ \\
\hline \hline
\end{tabular}

turbulent diffusion may be important [4,5]. Understanding the hydrodynamic stability of rotating stratified shear flows is critical to answering this question. In a linear inviscid analysis Molemaker et al. [6] showed that the introduction of weak stratification in a rotating shear flow leads to the growth of a purely hydrodynamic instability. Dubrulle et al. [7] extended this work into the astrophysical context as another possible mechanism for angular momentum transport in accretion disks.

We examine instability of flow between vertical concentric corotating cylinders with a stable axial density gradient. Table I gives values for the parameters defining the problem in our experiment, and Table II compares the parameter ranges in several previous laboratory experiments with our experiment. Our work extends the Reynolds number range and density gradient range of of the laboratory studies of the SRI (Table II).

This paper is organized as follows: Sec. II describes previous experimental and theoretical work, and Sec. III describes our experiment and data analysis. Section IV presents our results for the onset and characterization of the SRI instability and presents observations of a new flow state that mixes the background stratification. Section V discusses the results and considers the relevance of our experiments as a model of an accretion disk.

\section{BACKGROUND}

Experimental evidence for hydrodynamic angular momentum transport in rotating shear flows comes primarily from studies of turbulent flow of a fluid of uniform density contained between rotating cylinders. High Reynolds number $\left(\operatorname{Re} \sim 10^{6}\right)$ Taylor-Couette experiments have found angular momentum transport at rates necessary to allow gravitational collapse within a disk if extrapolated to astrophysical scales [3]. However, finite size effects from the top and bottom lids on the cylinders confound the results, making these experiments a poor model for accretion disks [2,12-14].

TABLE II. Comparison of experiments on the stratorational instability.

\begin{tabular}{lcccccc}
\hline \hline Experiment & $\eta$ & $\Gamma$ & $\mu$ range & $\operatorname{Re}_{i}$ & $N(\mathrm{rad} / \mathrm{s})$ & $\mathrm{Fr}$ \\
\hline Refs. [16,17] & 0.77 & 48 & 0 & $100-1800$ & $0.68,0.82,0.88,0.93$ & $0.2-5$ \\
Ref. [10] & 0.77 & 48 & $-0.5-1$ & $72-4030$ & $0,0.54,0.89,1.21$ & $0-1.45$ \\
Ref. [11] & 0.80 & 12 & $0-1$ & $339-1210$ & $0.85-3.1$ & 0.5 \\
Present experiment & 0.877 & 43.4 & $0-1$ & $100-14000$ & $1.57,3.14,4.71$ & $0.4-4$ \\
\hline \hline
\end{tabular}


Dubrulle et al. [7] emphasize that rotation and shear are not the only important hydrodynamic properties of accretion disks. Protostellar accretion disks are gravitationally bound, and photoevaporation by the protostar heats the outer layers of a disk, causing axial variations in density [1]. The effects of stratification in accretion disks are often neglected in theory and in experiments under the assumption that stratification stabilizes the fluid. This assumption was supported by early stratified Taylor-Couette experiments [15-17], which found that a stable axial density stratification stabilizes the flow with respect to centrifugal instabilities. However, a 1997 study by Boubnov and Hopfinger [10] found that for corotating cylinders a gravitationally stable density variation can be destabilizing.

An inviscid analysis by Molemaker et al. [6] shows that when both cylinders are rotating, stratified laminar base flow destabilizes through the growth of a linear instability. Dubrulle et al. [7] used both inviscid linear stability analysis and a WKB method to show that this instability, which they named the stratorotational instability (SRI), can be present in accretion disks and therefore may contribute to angular momentum transport in these disks. Shalybkov and Rüdiger [18] followed with a viscous theoretical study that hypothesized a new stability criterion for the stratified Taylor-Couette flow.

For a fluid of uniform density the well-known Rayleigh criterion for stability of inviscid rotating shear flows is [19]

$$
\frac{d\left(\Omega r^{2}\right)^{2}}{d r}>0
$$

where $\Omega(r)$ is the radially varying rotation rate of the shear flow and $r$ is the radial coordinate. For the concentric cylinder system the Rayleigh criterion becomes $\mu>\eta^{2}$, where $\mu$ is the ratio of the cylinder rotation rates, $\Omega_{o} / \Omega_{i}$, and $\eta$ is the ratio of the cylinder radii, $r_{i} / r_{o}$ [20]. Shalybkov and Rüdiger [18] proposed an analogous criterion for stability in a stratified rotating shear flow,

$$
\frac{d(\Omega r)^{2}}{d r}>0,
$$

which can be written more compactly as $\mu>\eta$.

The first detailed experiment on the SRI in a system with independently rotating cylinders was by Le Bars and Le Gal [11], who confirmed the $\mu>\eta$ stability criterion over the range of Reynolds numbers they explored (Table I). The experiments in Ref. [11] and the simulations in Ref. [18] examined the inner Reynolds number range $0<\mathrm{Re}_{i}<1200$. The measurements in Ref. [11] were for a fixed internal Froude number, $\mathrm{Fr}=0.5$. Dubrulle et al. [7] estimate that $\mathrm{Fr} \sim 3$ for an accretion disk; they found that large $N$ stabilizes flow beyond the stability onset of Taylor vortex flow, while for small $N$ the flow becomes unstable below the onset of the Taylor vortex flow instability.

This paper expands the range of density gradients examined in experiments by a factor of two and is the first to examine how the axial wavelength of the instability depends on Fr. The Reynolds number range investigated in the present work extends to 14000 , which is more than three times the maximum Reynolds number (4030) of previous experiments on the SRI (Table II).

\section{METHODS}

The parameters for our Taylor-Couette system [Fig. 1(a)] are given in Table I. The cylinders rotate independently, driven by computer-controlled stepper motors.

\section{A. Stratification}

The data presented here are for three fixed values of $N: 1.57,3.14$, and $4.71 \mathrm{~s}^{-1}$. Large $N$ is not accessible for large aspect ratios $(h \gg d)$ when using $\mathrm{NaCl}$ solutions, which have a maximum density of $1200 \mathrm{~kg} / \mathrm{m}^{3}$ (hence a maximum density range of $20 \%$ ). Our experiments use sodium polytungstate salt $\left(\mathrm{Na}_{6} \mathrm{H}_{2} \mathrm{~W}_{12} \mathrm{O}_{4}\right)$ solutions, which have a maximum density greater than $3000 \mathrm{~kg} / \mathrm{m}^{3}$ [21]; thus 

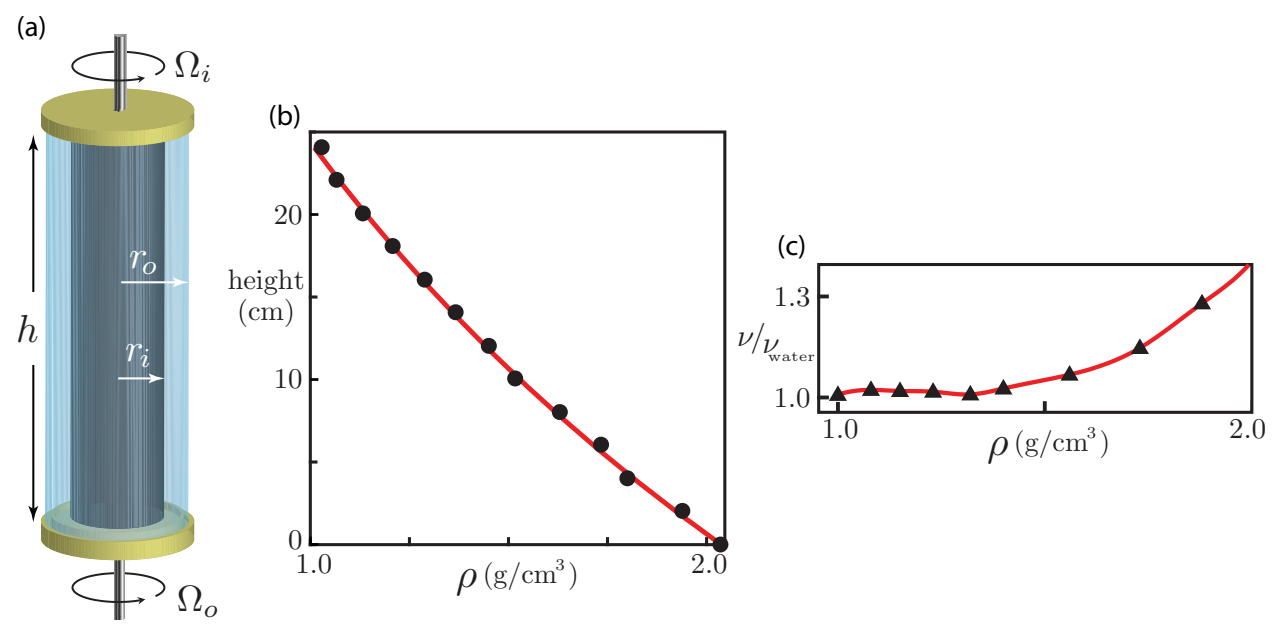

FIG. 1. (a) A schematic diagram of the experimental system. (b) Density as a function of height for $N=5.3 \mathrm{~s}^{-1}$. The black circles are measurements, and the solid red curve is a fit of the data to an exponential. (c) Viscosity of an aqueous solution of sodium polytungstate salt, as a function of density. Black triangles are the manufacturer's data for the kinematic viscosity $v$ at $25^{\circ} \mathrm{C}$ [21] normalized by the viscosity of water $v_{\text {water }}$. The solid red curve is a cubic spline fit to the manufacturer's data.

the density of the salt solutions can vary by a factor of three. We used a maximum density variation of a factor of two because at higher densities the viscosity increases rapidly with density.

We use two computer-controlled syringe pumps to create a stable vertical stratification in the gap. One syringe contains fresh water and the other contains an aqueous solution of sodium polytungstate along with a small amount $(<1 \%)$ of Kalliroscope flakes for visualization. A control algorithm calculates the pump rates necessary to create an exponentially varying density $\rho(z)$ [Fig. 1(b)]. We use an exponential density gradient to have a constant buoyancy frequency $N$.

We determine the density stratification by removing approximately $1 \mathrm{ml}$ of fluid at height increments of $2 \mathrm{~cm}$ using a syringe with a long needle mounted to a translation stage driven by a stepper motor. The extracted fluid is analyzed with an Anton-Paar density meter, which is accurate to $\pm 1 \mathrm{~kg} / \mathrm{m}^{3}$. The fluid sample is then reinjected into the system to minimize the effects of the measurement.

The viscosity of sodium polytungstate aqueous solutions depends on the salt concentration and therefore the varies as a function of height. We obtain the viscosity as a function of height using a cubic spline fit to the viscosity values given by the manufacturer [21] [Fig. 1(c)]. We compute the Reynolds numbers using the average kinematic viscosity value in the middle third of the system, which is the region that we analyze. Over this region the viscosity varies at most $\pm 2.2 \%$ for the largest stratification we use, and the variation with height is less than $1 \%$ for the other stratifications.

The buoyancy frequency is calculated by fitting an exponential curve to the density measurements. An example density profile is given in Fig. 1(b). The density is accurately exponential except near the ends of the annulus, where the deviations from an exponential can become pronounced due to Ekman cells. However, these deviations do not significantly affect the stratification in the central region that we analyze.

\section{B. Determining onset of instability}

After preparing the background stratification, we determine the instability boundary by rotating the cylinders in solid body rotation $(\mu=1)$ and then slowly decreasing $\operatorname{Re}_{o}$ while $\operatorname{Re}_{i}$ is held fixed. The instability onset is determined to within $1 \%$ of $\mathrm{Re}_{o}$ by stepping back and forth through the 


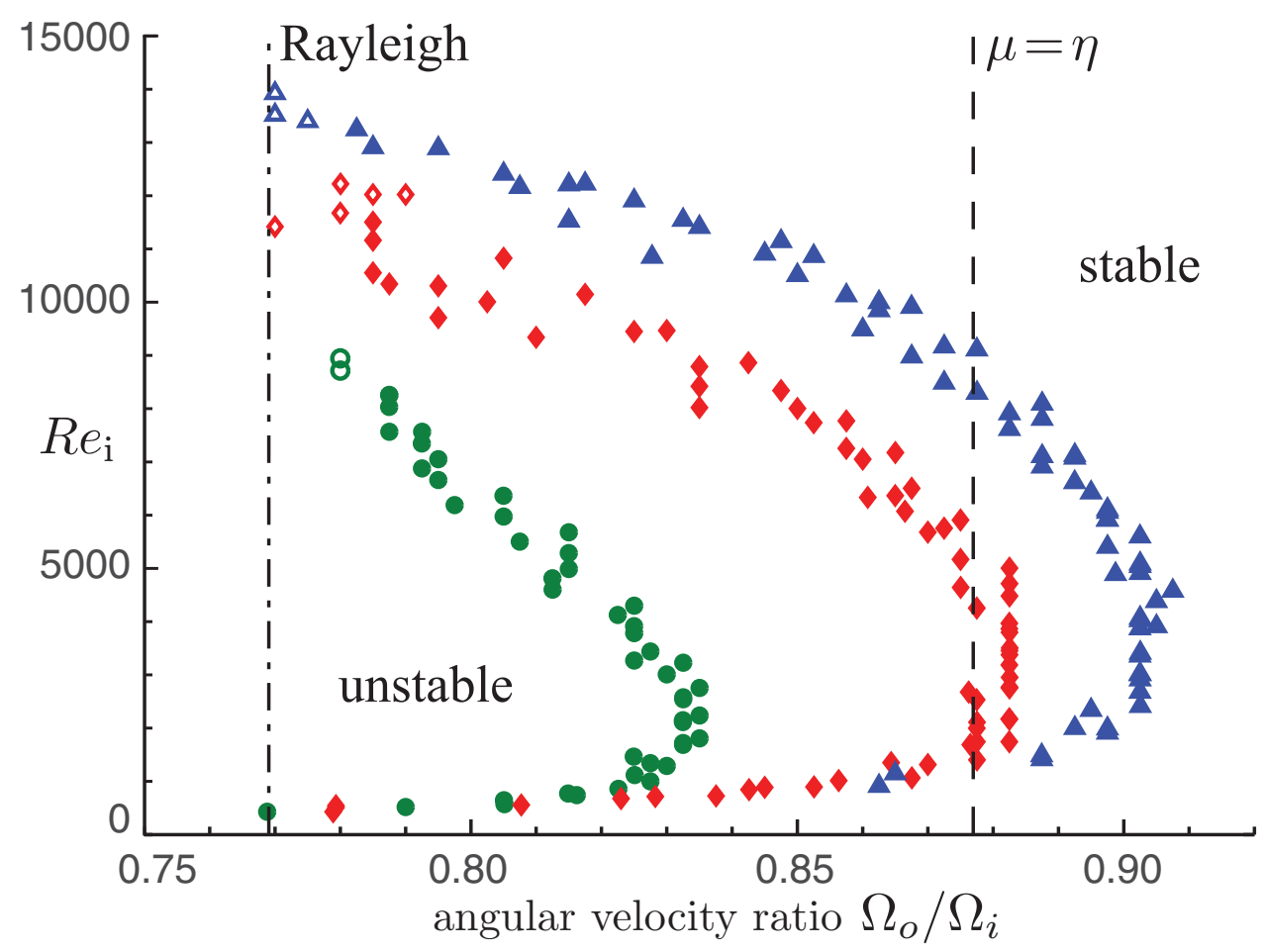

FIG. 2. Observations of the SRI onset for increasing anticyclonic shear as $\mu=\Omega_{o} / \Omega_{i}$ was decreased from solid body rotation $\left(\mu=1\right.$ ) and $\operatorname{Re}_{i}$ was held fixed (green circles, $N=1.57 \mathrm{~s}^{-1}$; red diamonds, $N=3.14 \mathrm{~s}^{-1}$; blue triangles, $N=4.71 \mathrm{~s}^{-1}$ ). For all three values of $N$ examined the SRI flow was observed in the region to the right of the vertical dot-dash $\mu=\eta^{2}$ line where stability is predicted by Rayleigh's criterion for an inviscid unstratified fluid [19]. The open symbols near the Rayleigh line identify conditions under which the base state became unstable to a nonperiodic state rather than to the SRI. For a viscous stratified fluid Shalybkov and Rüdiger [18] predict stability to the right of the dashed $\mu=\eta$ line, while our experiments with $N=4.71 \mathrm{~s}^{-1}$ exhibit the SRI in that region.

instability boundary in a binary search. Measurements of the axial wavelength of the stratorotational state as a function of inner Froude number, $\mathrm{Fr} \equiv \Omega_{i} / N$, are made by varying $\Omega_{i}$ for each of the three values of $N$.

As noted in Ref. [11], the SRI does not mix the fluid even if the stratorotational state is observed for very long times. We conducted extensive measurements for periods even longer than one day and found that the density stratification did not change significantly, except at the ends, which showed mixing due to Ekman circulation. The Ekman cells were typically $3 \mathrm{~cm}$ or less high, but at the largest $\operatorname{Re}_{i}, 14000$, the Ekman cells were about $7 \mathrm{~cm}$ high at $N=1.57 \mathrm{~s}^{-1}$.

\section{Spatial and temporal frequencies}

We analyze the spatiotemporal characteristics of the pattern by capturing images using an Edgertronic high speed monochrome camera and/or a Nikon D200 digital still camera. We analyze the time series intensity data for a narrow vertical strip in the central region of the digital image. The power spectral density is found using the fast Fourier transform function in Matlab (Fig. 4).

We determine the axial wavelength by measuring the distance in pixels between successive vortex pairs in a still image (Fig. 5). The length in pixels is converted into centimeters by imaging a ruler near the region being analyzed. 

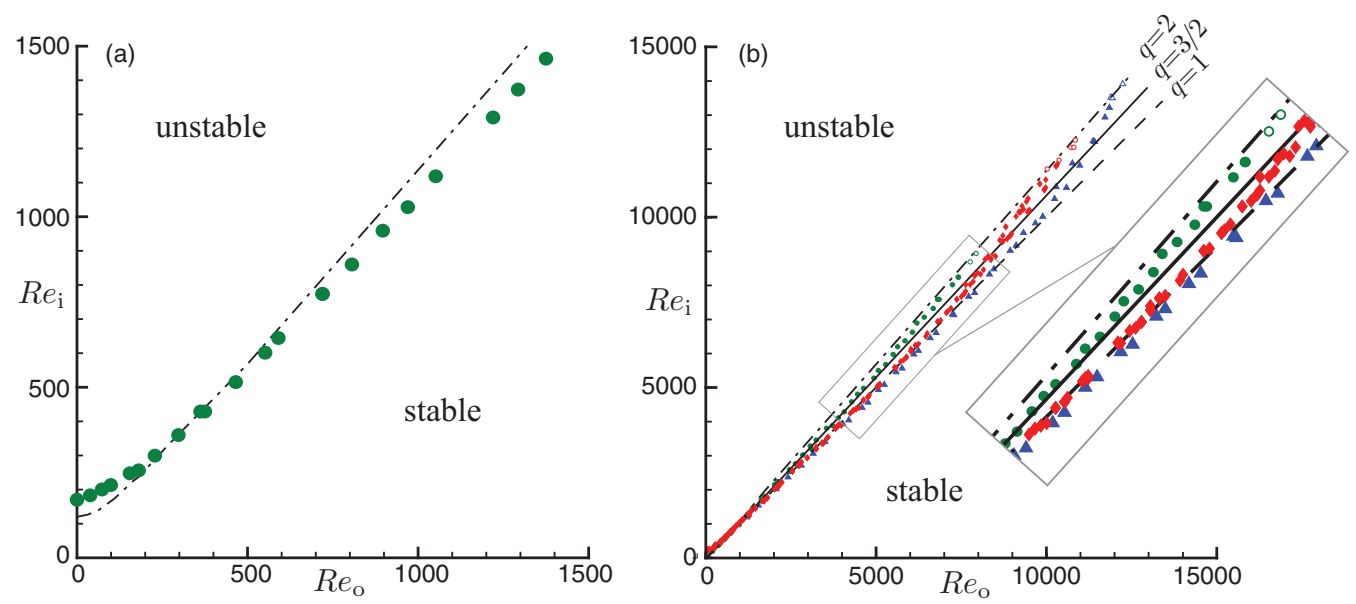

FIG. 3. (a) At low Reynolds numbers $\operatorname{Re}_{i}$ and $\mathrm{Re}_{o}$ the vertical density gradient stabilizes the laminar flow compared with the curve for the unstratified Taylor vortex flow instability (dot-dash curve), but at $\operatorname{Re}_{i}$ and $\operatorname{Re}_{o}$ above about 350 a density gradient destabilizes the laminar flow before the onset of Taylor vortex flow. (b) For $\mathrm{Re}_{i}$ and $\mathrm{Re}_{o}$ an order of magnitude larger than in (a) the laminar flow is destabilized more for a larger density gradient, but the effect of stratification decreases at higher Reynolds numbers (green circles, $N=1.57 \mathrm{~s}^{-1}$; red diamonds, $N=3.14 \mathrm{~s}^{-1}$; blue triangles, $N=4.71 \mathrm{~s}^{-1}$ ). The open symbols near the Rayleigh line correspond to a nonperiodic state that mixes the fluid. The Taylor stability line [22] is the dot-dashed curve, which is indistinguishable from the Rayleigh line (labeled $q=2$ using the notation of Balbus and Hawley [1]) at high Reynolds numbers. The Keplerian velocity profile is the solid line $(q=3 / 2)$, and the dashed line $(q=1)$ is the Shalybkov and Rüdiger prediction for instability onset. The inset enlarges the region where the data cross the $\mu=\eta$ line. The perpendicular distance in Reynolds number between the $q=1$ and $q=2$ lines is at most 600 .

\section{RESULTS}

\section{A. Onset of the SRI}

We observe a supercritical Hopf bifurcation from the base flow to the SRI flow when $\mu$ is slowly decreased from rigid body rotation $(\mu=1)$, as shown in the stability diagram in Fig. 2 . This bifurcation was also observed in Ref. [11], which found in measurements for $\operatorname{Re}_{i}$ up to 1210 (Table II) that the base flow was stable for $\mu>\eta$, in accord with the prediction in Ref. [18]. However, our measurements with $\operatorname{Re}_{i}$ ranging up to 14000 reveal that for $N>3.1 \mathrm{~s}^{-1}$ the $\mu>\eta$ the stability criterion in Ref. [18] does not hold, as Fig. 2 illustrates. This observation of the failure of the $\mu=\eta$ stability limit is in accord with later numerical simulations by Rüdiger and Shalybkov [23].

The SRI onset data in Fig. 2 are replotted in Fig. 3 for comparison with the classic Taylor vortex flow instability of an unstratified fluid. The nonaxisymmetric SRI and the axisymmetric Taylor vortex flow instability onset curves fall close to one another, even for large $N$. At small $\operatorname{Re}_{o}$ a density gradient stabilizes the stratified fluid compared to the Taylor vortex flow, but for $\operatorname{Re}_{o} \gtrsim 350$ the SRI precedes the Taylor instability [Fig. 3(a)]. The destabilizing effects of stratification increase with Reynolds number until $\operatorname{Re}_{o} \gtrsim 7500$, where the onset of instability approaches the Taylor line [Fig. 3(b) inset]. The SRI occurs both above and below the line corresponding to a Keplerian velocity profile, where the $q$ parameter (Table I; see Ref. [1]) has the value 3/2. The SRI also occurs both above and below the $\mu=\eta(q=1)$ line, so there is no apparent relation of the instability to the value of $q$.

The time periodicity of the SRI is illustrated by the power spectral density graph in Fig. 4(a), which was computed from a time series of pixel intensities from a movie made using Kalliroscope to visualize the flow. The most intense spectral peak, $2 \pi f / \Omega_{a v}=5.941$, shows that the $m=6$ state 

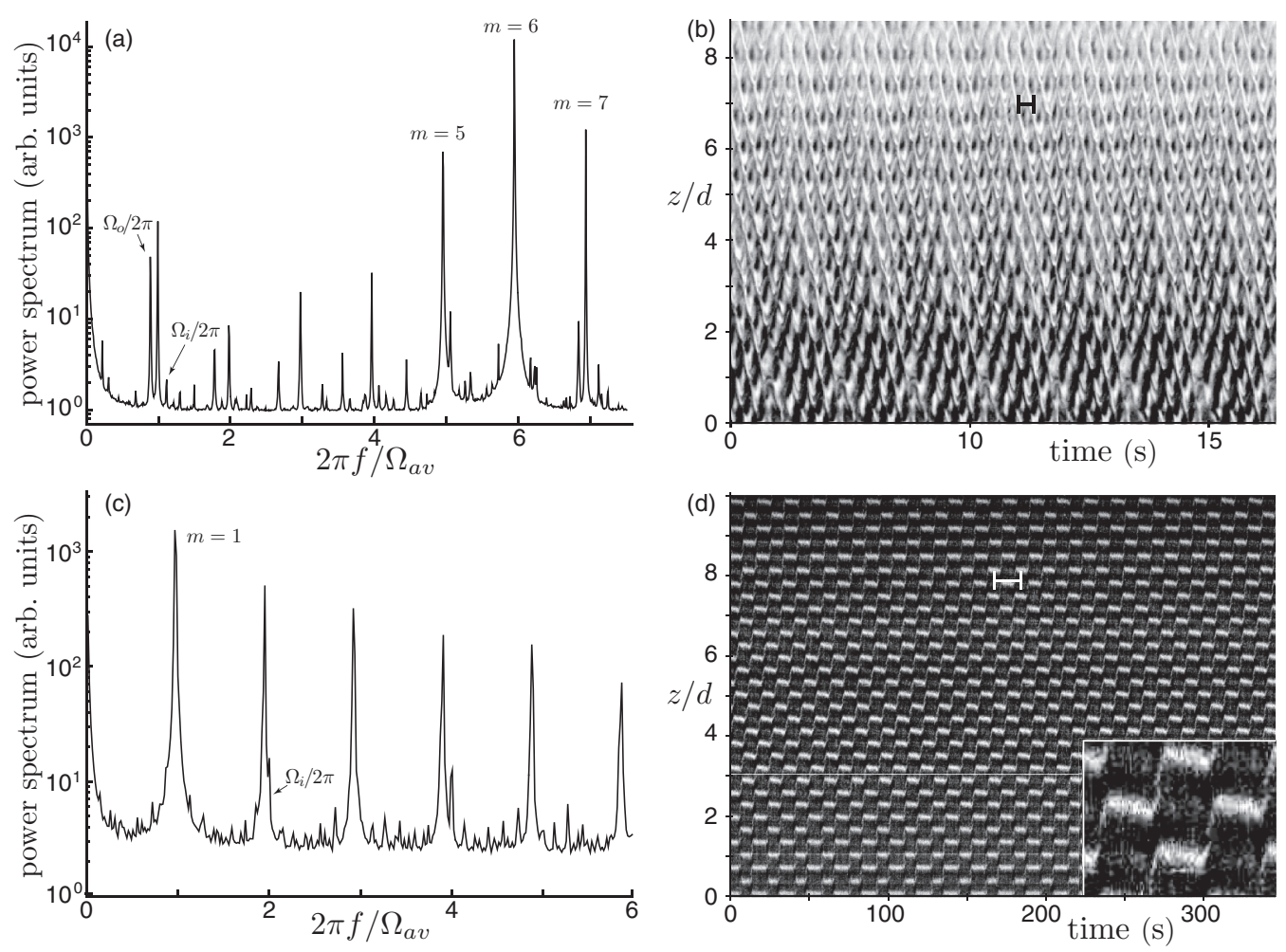

FIG. 4. (a) A power spectrum of a movie pixel time series for $\mu=0.80$ shows that the SRI flow is periodic. (b) The time evolution of a vertical strip of pixels reveals that the periodic state in (a) consists of interpenetrating spirals; the black horizontal bar shows one period $(0.374 \mathrm{~s})$ of an $m=6$ interpenetrating spiral, which corresponds to the dominant peak in the spectrum in (a), $2 \pi f / \Omega_{a v}=5.941$; strong peaks from the $m=5$ and $m=7$ states are also present. The low intensity peaks are harmonics and sums and differences of the cylinder and flow frequencies. The interpenetrating spirals rotate at approximately the average rotation rate $\left(\Omega_{a v} / 2 \pi=0.45 \mathrm{~Hz}\right)$ of the inner and outer cylinders $\left(\Omega_{i} / 2 \pi=0.5 \mathrm{~Hz}\right.$ and $\left.\Omega_{o} / 2 \pi=0.4 \mathrm{~Hz}\right)$. The axial wavelength in the image in (b) is $\lambda / d=2.38$ and is plotted as an open green circle in Fig. 6. [In (a) and (b) $N=1.9 \mathrm{~s}^{-1}, \mathrm{Re}_{i}=571, \mathrm{Re}_{o}=815$, and internal $\mathrm{Fr}=1.32$.] (c) A power spectrum of a movie pixel time series for the $\mu=0$ (outer cylinder at rest) SRI state shows that it is also periodic. The six harmonics yield a fundamental frequency $2 \pi f / \Omega_{a v}=0.9765$. (d) An image sequence showing the time evolution of a vertical strip from a movie of the flow state in (c); the white bar shows the period (17.23 s) corresponding to the fundamental frequency in (c). The inset in (d) shows a section of the pattern with $400 \%$ magnification. The axial wavelength in the image is $\lambda / d=1.18$ and is plotted as an open green circle in Fig. 6 . The vertical distance between two white stripes, where one is directly above the other, is one half of the axial wavelength. [In (c) and (d) $N=1.57 \mathrm{~s}^{-1}, \Omega_{i} / 2 \pi=0.119 \mathrm{~Hz}, \operatorname{Re}_{o}=0, \operatorname{Re}_{i}=169$, and $\mathrm{Fr}=0.48$ (the lowest data point in Fig. 3(a)).] Both sets of data were taken within $3 \%$ of the onset of the SRI states.

is dominant and that the pattern rotates approximately at the average rotation rate of the inner and outer cylinders, as previously observed in Ref. [11].

The spatial pattern constructed from a time series of vertical strips from the movie reveals that the SRI flow consists of interpenetrating spirals [Fig. 4(b)], as found in Ref. [11] [our Fig. 4(b) is similar to those authors' Fig. 3(a)].

While our focus is on instability of flow for $\mu \sim 1$, as shown in the stability diagram in Fig. 2, we also examined flow instability with the outer cylinder at rest, $\mu=0$, as studied in Ref. [16] using Kalliroscope visualization and in Ref. [17] using dye measurements. Those previous observations 

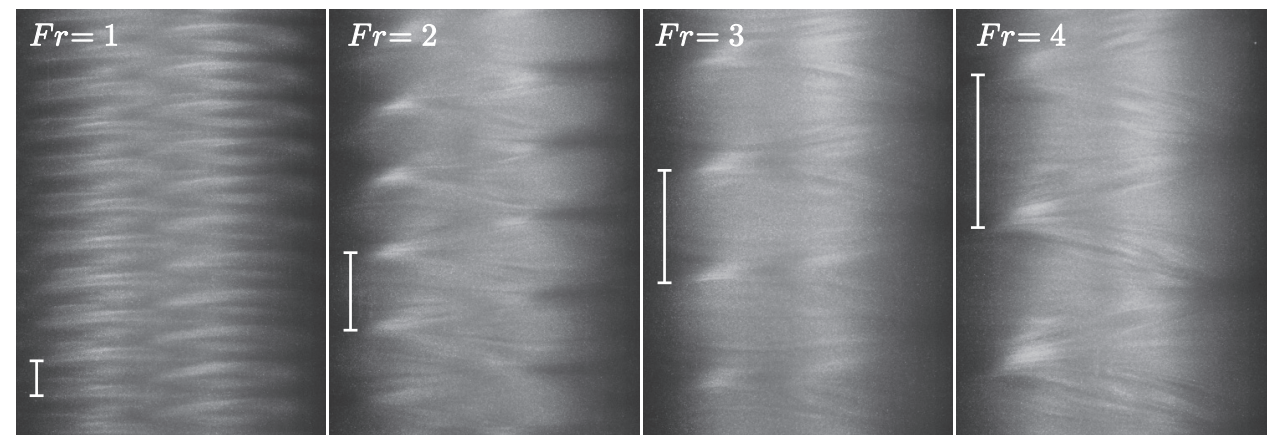

FIG. 5. Images of the SRI pattern for four values of internal Froude number $\mathrm{Fr}=\Omega_{i} / N$ with $N=1.57 \mathrm{~s}^{-1}$. The wavelengths determined from the images are plotted in Fig. 6. The length of the smallest scale bar is $\lambda=1.81 d$, which is $1.07 \mathrm{~cm}$. ( $\operatorname{Re}_{i}=419,838,1257,1675$ and $\mu=0.768,0.823,0.830,0.835$, respectively. $)$

are supported by our experiments using Kalliroscope visualization: Fig. 4(c) shows a power spectrum and Fig. 4(d) shows the space-time evolution of the flow. The power spectrum shows that this SRI state is also periodic, and the fundamental frequency is also nearly equal to the average rotation rate, as observed in Ref. [17]. However, as Fig. 4(d) illustrates, the flow pattern is not interpenetrating spirals, but the flow is spatially periodic in the axial direction.

The two distinct flow states at onset of the SRI were predicted in Ref. [18]: "Depending on the value of $\mu$ and $\eta$ this new regime [the SRI] can be either oscillating and axisymmetric or nonaxisymmetric and azimuthally drifting." A more detailed experimental characterization of the secondary flow than provided in Fig. 4 will require information on the time evolution of the fluid motion within the annulus, which could be obtained from movies made with slit illumination of flow in the gap between the cylinders.

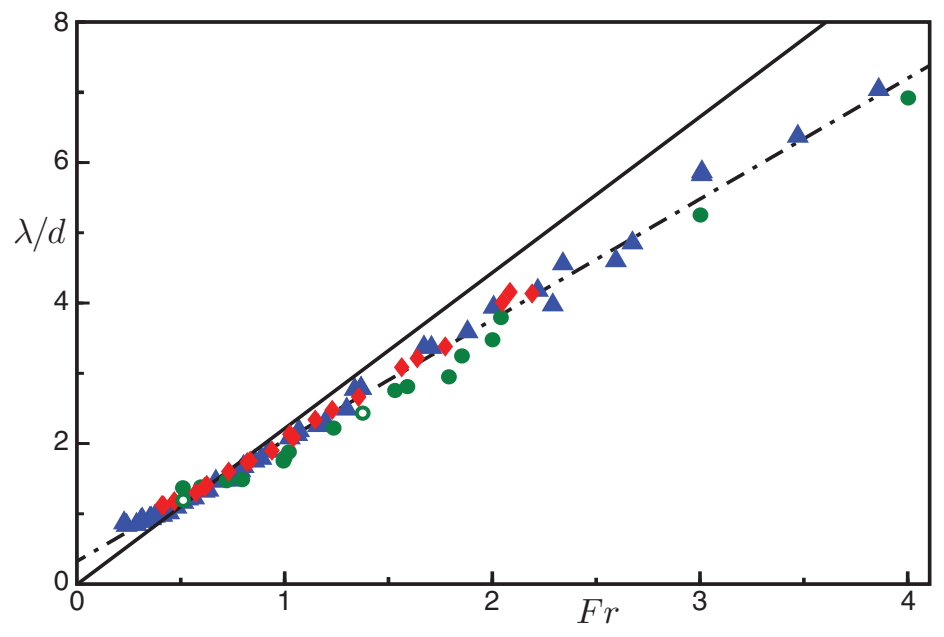

FIG. 6. Axial wavelength $\lambda$ at the onset of the SRI, normalized by the gap width $d$, as a function of $\mathrm{Fr}=\Omega_{i} / N$. The symbols are the same as in Figs. 2 and 3 (green circles $N=1.57 \mathrm{~s}^{-1}$; red diamonds, $N=3.14 \mathrm{~s}^{-1}$; blue triangles, $N=4.71 \mathrm{~s}^{-1}$ ). A linear fit to the data (the black dash-dot line) yields slope $=1.72$ and intercept $=0.32$; our results differ from the slope value 2.2 and intercept value zero predicted by linear inviscid theory (solid line) [Eq. (3)] [6]. The open green circles are the wavelengths from the images in Fig. 4(b), $\lambda / d=2.38$, and Fig. $4(\mathrm{~d}), \lambda / d=1.18$. 

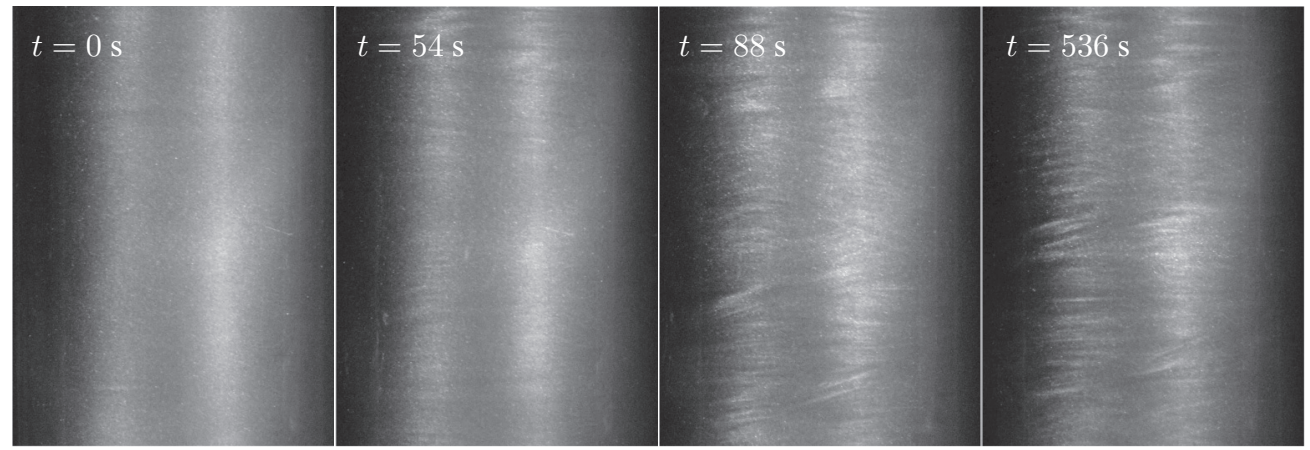

FIG. 7. Images showing the development of a spatially nonperiodic state from laminar flow at $\operatorname{Re}_{i}=9000$ during a 9 min period after the ratio of the cylinder rotation rates had been reduced from $\mu=0.79$ to $\mu=0.78$ $\left(N=1.57 \mathrm{~s}^{-1}\right)$.

\section{B. Wavelength dependence on internal Froude number}

We have made the first investigation of the dependence of the axial wavelength $\lambda$ on the internal Froude number Fr, as illustrated by images at four values of Fr in Fig. 5. We compare our wavelength measurements with the inviscid analysis in Ref. [6], which found

$$
\lambda / d=(2 \pi \operatorname{Ro} \sqrt{\operatorname{Ro}+1}) \mathrm{Fr},
$$

where the Rossby number Ro defined by their Eq. (4), is approximately Ro $=-0.75$ in our work. The prediction in Ref. [6] for the slope is compared with our measurements in the caption of Fig. 6. Beyond the onset of SRI, we find little change in the wavelength.

\section{High Reynolds number}

At Reynolds numbers greater than 2000, above the range examined by in Ref. [11], the instability curves in a plot of $\operatorname{Re}_{i}$ vs $\mu$ turn upward (Fig. 2). For $\operatorname{Re}_{i}$ larger than about 5000, the SRI occurs for lower values of $\mu$; thus larger global rotation stabilizes the fluid with respect to the SRI. This
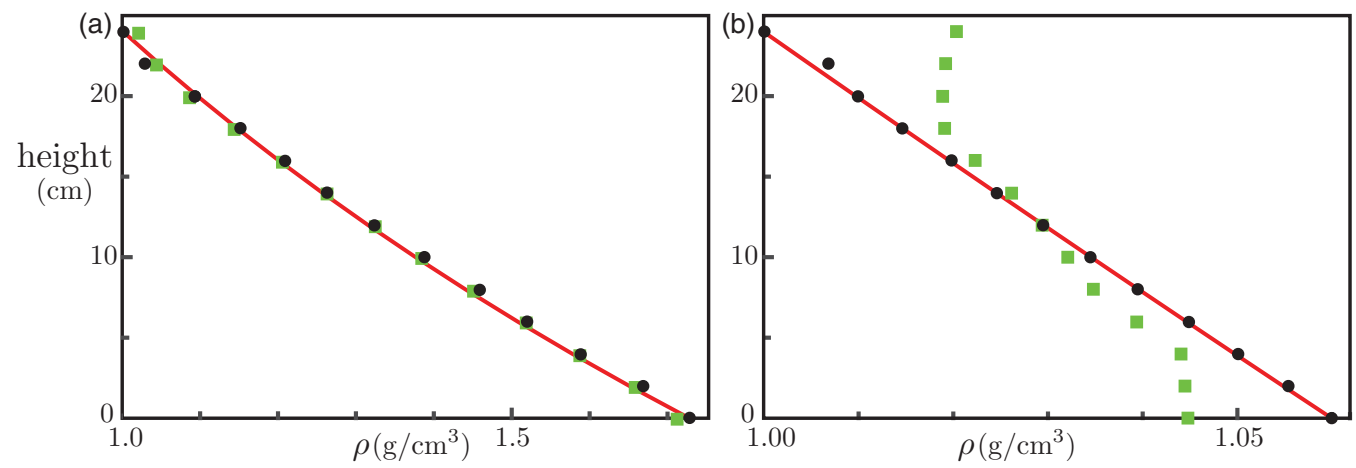

FIG. 8. (a) Density measurements (black circles) with an exponential fit (red curve) for a state with a 73\% increase in density $\left(N=4.71 \mathrm{~s}^{-1}\right)$ from the top to the bottom of the fluid. The green squares are the density measurements taken after the SRI was observed for more than 30 min over a 2 day period; thus the SRI does very little mixing even when the density variation is large. (b) Density measurements (black circles) with an exponential fit (red curve) before the fluid transitions from the laminar base state to a nonperiodic state for a fluid with a $6 \%$ change in density $\left(N=1.57 \mathrm{~s}^{-1}\right)$. The fluid mixes much more rapidly for the noperiodic state (Fig. 7); green squares in the graph show the density 9 min after the transition to the nonperiodic state. 
behavior is not predicted by existent SRI theory and was not reported in previous experiments or in computational studies.

For large $\mathrm{Re}_{i}$, instability of the base state leads not to the SRI but to a spatially nonperiodic state, which rapidly mixes the fluid, as illustrated by the images in Fig. 7 and shown by open symbols in the stability diagrams of Figs. 2 and 3(b). Larger stratification suppresses the onset of this nonperiodic state (Fig. 3). The characteristic spiral modes of the SRI are not present in the nonperiodic state, although large-scale and fine-scale structure is present, as can be seen in Fig. 7. The mixing in the spatially nonperiodic state reduces the density stratification, as Fig. 8 illustrates. A spectral analysis of the nonperiodic state reveals the same fundamental frequency and harmonics as the SRI because the large-scale structure rotates at the average speed of the two cylinders, but the mixing at small scales increases the background noise level in the power spectra.

The nonperiodic state appeared at a fairly well-defined value of $\mu$ as it was decreased in small steps $(\Delta \mu=0.01)$. It was not possible to determine whether there was hysteresis in the onset of the nonperiodic state because the fluid mixed rapidly once the nonperiodic state appeared. Consequently, we were unable to cross back and forth from the laminar to the nonperiodic state in the same way as was done in determining the onset of the SRI state.

We examined the criterion of Richard and Zahn [24], who define a critical gradient Reynolds number, $\mathrm{Re}^{*}$, for the onset of subcritical instability in a uniform fluid:

$$
\mathrm{Re}^{*} \equiv \frac{r_{m}^{3}}{v} \frac{\left|\Omega_{i}-\Omega_{o}\right|}{d} \gtrsim 6 \times 10^{5},
$$

where $r_{m}$ is the mean value of the inner and outer radii. The gradient Reynolds numbers at which we observed the nonperiodic state were $\mathrm{Re}^{*} \geqslant 6.7 \times 10^{5}$, which satisfies the condition in Eq. (4).

\section{DISCUSSION}

Studies of the stability of a stratified fluid between rotating concentric cylinders have been motivated by the possibility that this system might provide insight into fluid instability of astrophysical accretion disks. The experiments have used vertical corotating cylinders with an axial density gradient. In order to impose an anticyclonic shear as in accretion disks, the outer cylinder is rotated more slowly than the inner cylinder.

The experiments have revealed a supercritical Hopf bifurcation from the base flow to the "stratorotational instability" (SRI) [10,11,15-17]. For $\mu>0$ the secondary is flow is interpenetrating spirals [Fig. 4(b)]. For $\mu \approx 0$ the secondary flow is also temporally and spatially periodic [Fig. 4(d)], but the pattern appears different.

The present experiment extends by a factor of two the maximum axial density gradient examined and by a factor of three the maximum Reynolds number investigated for the SRI (Table II). At large Reynolds numbers we find the primary instability is not the SRI but rather a nonperiodic flow that rapidly mixes the fluid (Fig. 7). Thus it is unlikely that the SRI is relevant at the far higher Reynolds numbers of protoplanetary disks. However, the effect of axial density stratification on protoplanetary disks may be important through other instabilities such as the subcritical instability proposed by Marcus et al. [5,25].

For the two larger density gradients we have studied, $N=3.14$ and $4.71 \mathrm{~s}^{-1}$, we have found a range of $\mathrm{Re}_{i}$ where the instability occurs for $\Omega_{o} / \Omega_{i}>r_{i} / r_{o}$ (Fig. 2), contrary to the prediction of Ref. [18] but consistent with later numerical simulations [23].

While there is a striking difference between the curves for different $N$ in Fig. 2, the dependence of SRI onset on stratification is much less striking in the Taylor-like graph of $\operatorname{Re}_{i}$ vs $\operatorname{Re}_{o}$ (Fig. 3). The symmetry of the emergent SRI differs from axisymmetric Taylor vortex flow of an unstratified fluid, yet the SRI and Taylor vortex flow instability curves are surprisingly close. The smallest and largest density gradients that we examined differ by a factor of nine, but the corresponding instability curves differ in Reynolds number by at most 9\% (Fig. 3). For small $\operatorname{Re}_{o}$, the onset of the SRI occurs 
for $\mathrm{Re}_{i}$ larger than for the axisymmetric Taylor vortex flow, while for $\mathrm{Re}_{o} \gtrsim 350$ the onset of SRI occurs for smaller Reynolds numbers than the onset of Taylor vortex flow.

We have found a strong dependence of the axial wavelength of the SRI flow on the internal Froude number Fr $=\Omega_{i} / N$ (Fig. 6). The wavelength increases linearly from the smallest measured value $(0.88 d)$ to the largest $(7 d)$, while in contrast the wavelength at onset of instability in the unstratified case is $2 d$ [22]. Given the large density gradient, we presume that end effects do not significantly affect the SRI flow.

At large $\operatorname{Re}_{i}$ (about 9000 for $N=1.57 \mathrm{~s}^{-1}$ and 14000 for $N=4.71 \mathrm{~s}^{-1}$ ), the instability of the base flow that occurs with increasing anticyclonic shear (decreasing $\Omega_{o} / \Omega_{i}$ ) leads to nonperiodic flow rather than the periodic SRI. In Taylor-type graphs of $\mathrm{Re}_{i}$ vs $\operatorname{Re}_{o}$, the SRI onset line is close to the Taylor vortex onset line in an unstratified fluid, where the radial dependence of the velocity for the azimuthal flow is close to the Rayleigh profile [19], $\Omega_{\phi}(r) \propto r^{-2}(q=2$, Fig. 3), rather than to the approximate Keplerian profile $\Omega_{\phi}(r) \propto r^{-3 / 2}(q=3 / 2)$ of protoplanetary disks.

\section{ACKNOWLEDGMENTS}

We thank Hepeng Zhang, Abhay Argarwal, Evander Harris, Shuqi Liu, John Scelzi, and Deon Rodenborn for their assistance. This research was supported in part by the Sid W. Richardson Foundation.

[1] S. A. Balbus and J. F. Hawley, Instability, turbulence, and enhanced transport in accretion disks, Rev. Mod. Phys. 70, 1 (1998).

[2] M. J. Burin, E. Schartman, and H. Ji, Local measurements of turbulent angular momentum transport in circular Couette flow, Exp. Fluids 48, 763 (2010).

[3] M. S. Paoletti, D. P. M. van Gils, B. Dubrulle, C. Sun, D. Lohse, and D. P. Lathrop, Angular momentum transport and turbulence in laboratory models of Keplerian flows, Astron. Astrophys. 547, A64 (2012).

[4] N. I. Shakura and R. Sunyaev, Black holes in binary systems. Observational appearance, Astron. Astrophys. 24, 337 (1973).

[5] P. S. Marcus, S. Pei, C.-H. Jiang, J. A. Barranco, P. Hassanzadeh, and D. Lecoanet, Zombie vortex instability. I. A purely hydrodynamic instability to resurrect the dead zones of protoplanetary disks, Astrophys. J. 808, 87 (2015).

[6] M. J. Molemaker, J. C. McWilliams, and I. Yavneh, Instability and Equilibration of Centrifugally Stable Stratified Taylor-Couette Flow, Phys. Rev. Lett. 86, 5270 (2001).

[7] B. Dubrulle, L. Marie, C. Normand, D. Richard, F. Hersant, and J. P. Zahn, An hydrodynamic shear instability in stratified disks, Astron. Astrophys. 429, 1 (2005).

[8] G. R. Spedding, The evolution of initially turbulent bluff-body wakes at high internal Froude number, J. Fluid Mech. 337, 283 (1997).

[9] S. Sparnocchia, G. Gasparini, M. Astraldi, M. Borghini, and P. Pistek, Dynamics and mixing of the Eastern Mediterranean outflow in the Tyrrhenian basin, J. Marine Syst. 20, 301 (1999).

[10] B. Boubnov and E. Hopfinger, Stability of the Couette flow between two independently rotating cylinders in a stratified fluid, Phys.-Dokl. 42, 312 (1997).

[11] M. Le Bars and P. Le Gal, Experimental Analysis of the Stratorotational Instability in a Cylindrical Couette Flow, Phys. Rev. Lett. 99, 064502 (2007).

[12] M. Avila, Stability and Angular-Momentum Transport of Fluid Flows Between Corotating Cylinders, Phys. Rev. Lett. 108, 124501 (2012).

[13] F. Nordsiek, S. G. Huisman, R. C. A. van der Veen, C. Sun, D. Lohse, and D. P. Lathrop, Azimuthal velocity profiles in Rayleigh-stable Taylor-Couette flow and implied axial angular momentum transport, J. Fluid Mech. 774, 342 (2015). 
[14] E. M. Edlund and H. Ji, Reynolds number scaling of the influence of boundary layers on the global behavior of laboratory quasi-Keplerian flows, Phys. Rev. E 92, 043005 (2015).

[15] E. M. Withjack and C. F. Chen, An experimental study of Couette instability of stratified fluids, J. Fluid Mech. 66, 725 (1974).

[16] B. M. Boubnov, E. B. Gledzer, and E. J. Hopfinger, Stratified circular couette-flow - instability and flow regimes, J. Fluid. Mech. 292, 333 (1995).

[17] F. Caton, B. Janiaud, and E. J. Hopfinger, Stability and bifurcations in stratified Taylor-Couette flow, J. Fluid. Mech. 419, 93 (2000).

[18] D. Shalybkov and G. Rüdiger, Stability of density-stratified viscous Taylor-Couette flows, Astron. Astrophys. 438, 411 (2005).

[19] Lord Rayleigh, On the stability, or instability, of certain fluid motions, Proc. London Math. Soc. 11, 57 (1880).

[20] S. Chandrasekhar, Hydrodynamic and Hydromagnetic Stability (Dover, New York, 1961).

[21] http://geoliquids.com (2016).

[22] G. I. Taylor, The stability of viscous liquid contained between two cylinders, Philos. Trans. R. Soc. London 223, 289 (1923).

[23] G. Rüdiger and D. A. Shalybkov, Stratorotational instability in MHD Taylor-Couette flows, Astron. Astrophys. 493, 375 (2009).

[24] D. Richard and J. P. Zahn, Turbulence in differentially rotating flows: What can be learned from the Couette-Taylor experiment, Astron. Astrophys. 347, 734 (1999).

[25] P. S. Marcus, S. Pei, C.-H. Jiang, and P. Hassanzadeh, Three-Dimensional Vortices Generated by SelfReplication in Stably Stratified Rotating Shear Flows, Phys. Rev. Lett. 111, 084501 (2013). 